\title{
Nel segno di Armida. Sul paratesto delle Lagrime del penitente di Angelo Grillo
}

\author{
Francesco Ferretti \\ Università di Bologna \\ francesco.ferretti@unibo.it
}

\section{Riassunto}

Il saggio si concentra sull'emblema spirituale che precede l'edizione napoletana (Stigliola, 1594) delle Lagrime del penitente di Angelo Grillo, formato da una crocefissione accompagnata dal distico: "Sani piaga di duol, piaga d'errore, / e diano gli occhi medicina al core". Di questo distico penitenziale si individua il modello in Gerusalemme liberata, XX 125, rilevando le differenti funzioni dell'arguzia nei due diversi contesti (Grillo sta introducendo una raccolta di parafrasi poetiche dei sette salmi penitenziali, laddove Tasso aveva rappresentato la disperazione di Armida innamorata di Rinaldo, vinta sul campo di battaglia e ormai prossima al suicidio). Dopodiché si considerano le successive apparizioni di questo "cartiglio" ecfrastico (scompagnato dall'immagine cristologica) e si analizzano alcune delle sue molteplici riprese e variazioni concettuali all'interno delle Lagrime e dei Pietosi affetti.

Parole chiave: Angelo Grillo; Torquato Tasso; Lagrime del penitente; Pietosi affetti; Gerusalemme liberata; emblematica; poesia ecfrastica.

\section{Abstract. Under the sign of Armida. About the paratext of Angelo Grillo's Lagrime del penitente}

This essay analyzes the spiritual emblema present in the first complete edition of Angelo Grillo's Lagrime del penitente (Naples, Stigliola, 1594): a crucifixion accompanied by the couplet: "Sani piaga di duol, piaga d'errore, / e diano gli occhi medicina al core". It is important not only to note the model of these verses, hitherto passed over in silence (Gerusalemme liberata, XX 125), but also to reflect on the different function of wit in the two different contexts: Grillo is introducing a collection of poetic paraphrases of the seven penitential psalms, while Tasso represented Armida’s desperation in love with Rinaldo, won on the battlefield and now close to suicide. Finally, the subsequent appearances of this ecphrastic epigraph are analyzed, separated from the original Christological image; and some of his multiple shots and conceptual variations are analyzed within the Lagrime and Pietosi affetti.

Keywords: Angelo Grillo; Torquato Tasso; Lagrime del penitente; Pietosi affetti; Gerusalemme liberata; emblematic forms; ecphrastic poetry. 
Il presente contributo costituisce un'integrazione all'analisi delle Lagrime del penitente di Angelo Grillo, già proposta in altra sede (Ferretti 2015). Non sarà questa l'occasione, dunque, per dilungarsi sul ruolo del monaco-poeta genovese, figura chiave per la messa a fuoco del gusto poetico in Italia tra Tasso e Marino, né sembra opportuno tornare a insistere sull'originalissima struttura del ciclo dei 138 sonetti improntati ai sette salmi penitenziali davidici ( $P_{s} 6$, $32,38,51,102,130,142)^{1}$ che il monaco licenzia in giovinezza (Grillo 1594, col precedente di un'edizione parziale in Grillo 1589 in appendice alle Rime spirituali, e di una autonoma e integrale, ma non ancora definitiva in Grillo 1593) e considererà sempre un 'satellite' delle Rime spirituali (edite e progressivamente accresciute tra il 1589 e il 1629; dal 1595 messe sotto il titolo di Pietosi affetti) ${ }^{2}$.

L'elemento sul quale si sente l'esigenza di aggiungere qualcosa rispetto a quanto già detto è l'emblema che accompagna la prima edizione autorizzata dall'autore delle Lagrime apparsa a Napoli presso Nicola Antonio Stigliola: ${ }^{3}$ un elemento paratestuale che precede il primo dei 138 sonetti e che consiste in una crocifissione accompagnata e, per così dire, chiosata da un distico a rima baciata da attribuire senzaltro allo stesso Grillo. Non si tratta di un'impresa, come si vede (fig. 1), perché la combinazione di testo e immagine non dà luogo a un "nodo di parole e di cose". ${ }^{4} \mathrm{Si}$ tratta, piuttosto, di una più libera composizione emblematica, visto che, da una parte, l'immagine cristologica ha la funzione simbolica di sviluppare in senso neotestamentario la materia veterotestamentaria del ciclo davidico; dall'altra il testo breve - ma non brevissimo, come imporrebbe il linguaggio dell'impresa - dà voce a quel penitente ideale chiamato a leggere le Lagrime identificandosi, a sua volta, nell'io lirico di Grillo, il quale, in quei testi, si propone come oratore universale, modello affettuoso di quella lacrimosa "pietà barocca" della quale il distico è pervaso:

Sani piaga di duol, piaga d'errore, e diano gli occhi medicina al core.

Per quanto concerne l'immagine cristologica giova ricordare che Grillo era già stato preceduto da altri nella presentazione del Calvario come punto di

1. Sulla fortuna delle parafrasi dei Salmi nel Cinquecento cfr. Quondam, 2005, pp. 189-192; Leri 2011 (ma si risalga anche a Leri, 1994, pp. 15-45); Zaja, 2013; Morace 2015; Pietrobon 2019.

2. Per la storia delle rime spirituali di Grillo, che si intreccia strettamente a quella delle Lagrime (edite insieme alla prima parte dei Pietosi affetti in varie occasioni: 1601, 1602, 1606, 1608, 1613), cfr. la Nota al testo di M. Chiarla in Grillo 2013, pp. 35-45 e Ferretti 2012, pp. 121-136. L'ottima ed. Grillo 2013 ripropone le cinque parti della raccolta secondo il testo definitivo Grillo 1629.

3. Editore che stampa anche due fondamentali scritti in prosa tassiani: i Discorsi del poema eroico e il Conte overo delle imprese. Sulla multiforme attività di Stigliola, cfr. Ricci 2019.

4. Cfr. Arbizzoni 2002 (la definizione che dà il titolo al volume è di Ammirato 1562, p. 10). L'emblematica rinascimentale, com'è noto, fiorisce a seguito del fortunatissimo Emblematum liber di Andea Alciato del 1531 (oggi riedito in Alciato 2009): su questo genere, oltre al classico Praz 2014 (volume del 1946 recentemente ristampato), si recuperi anche il meno noto Giraud 1982. 
riferimento per una meditazione di matrice davidica (si veda il Cristo in croce tra dolenti, nell'edizione 1572 della fortunata antologia giolitina di salmi poetici curata da Francesco Turchi, fig. 2) ${ }^{5}$ e che Grillo stesso avrebbe replicato questo tipo di iconografia collocando o facendo collocare in sede incipitaria immagini relative alla morte di Cristo al principio dei Pietosi affetti del 1595: sia nella princeps (Cristo in croce tra dolenti, fig. 3) sia nella riedizione del 1596 (Crocifissione, fig. 4). Per quanto concerne il distico, invece, ci si è limitati a segnalare, in precedenza, che esso sarebbe riapparso privo dell'immagine, a mo' di epigrafe, nell'edizione definitiva del 1629 (fig. 5), preceduto da due concettosissime "sospirose" "preparationi in prosa" che assolvono a una funzione complementare, sviluppando ulteriormente la vocazione "lacrimistica" connaturata alla raccolta di sonetti. ${ }^{6} \mathrm{Di}$ questo distico ecfrastico, tuttavia, non era stato finora individuato e discusso il modello, che coincide, sorprendentemente, con l'ott. 125 del canto XX della Gerusalemme liberata, nella quale Armida, novella Didone e novella Cleopatra, si accinge a darsi la morte con una delle frecce con le quali avrebbe voluto uccidere l'amato Rinaldo sul campo di battaglia attorno a Gerusalemme:

Dimostratevi in me (ch'io vi perdono

la passata viltà) forti ed acute.

Misera Armida, in qual fortuna or sono,

se sol da voi posso sperar salute?

Poi ch'ogn'altro rimedio è in me non buono

se non sol di ferute a le ferute,

sani piaga di stral piaga d'amore,

e sia la morte medicina al core. ${ }^{7}$

Il distico di Grillo, come si vede, è la riscrittura in senso spirituale del paradosso erotico espresso da Armida ai vv. 7-8 per mezzo di uno di quei fregi concettosi che caratterizzano il sublime della Liberata, uno di quei 'madrigalismi' intollerabili alle orecchie dei detrattori di Tasso (come ad esempio Galileo), che, trasgredendo apparentemente il dettato sublime e correggendolo in senso lirico, servono in realtà ad amplificarlo in senso patetico. ${ }^{8}$ La premessa della risoluzione suicida di Armida, infatti, è una sottigliezza di matrice petrarchesca, che allude al topos di Amore arciere ('dal momento che non c'è

5. Su questa fortunata antologia del 1568, che nel 1569 fu riedita e nel 1572 ebbe una seconda edizione, cfr. Zaja 2014; Morace 2018; Pietrobon 2019 passim. Di Morace si veda inoltre l'ed. critica della princeps: Turchi 2016. Si noti che, come rileva la studiosa (Turchi 2016, p. 47, n. 4; Morace 2018, p. 45 n. 4), nelle edd. 1568 e 1569 erano presenti invece due xilografie veterotestamentarie: rispettivamente una Betsabea al bagno e un David orante con la cetra ai piedi.

6. Sul genere lacrimistico post-tridentino (oltremodo eterogeneo e irriducibile a un solo paradigma, nonostante l'influenza delle Lagrime di san Pietro di Tansillo), cfr. Quondam 2005, pp. 192-196; Piatti 2007; Piatti 2010; Torre 2012; Ferretti 2012, pp. 77-79 e 169-174; Pietrobon 2019, pp. 110-114.

7. Si cita il testo Caretti da Tasso 2009.

8. Cfr. (anche per la bibliografia) Ferretti, 2019a. 
più speranza per me se non quella che sanare le ferite d'amore con le ferite inferte da armi reali'). Da questa premessa discende, secondo una logica di tipo arguto, il paradosso che impone il suicidio come remedium amoris, in virtù di una paradossale strategia 'omeopatica': 'la ferita materiale di queste frecce curi la ferita immateriale inferta dalla freccia di Cupido e sia la morte medicina per il cuore innamorato'.

Quello di Tasso, insomma, è l'elemento trasgressivo che contamina con fregi lirici il sublime che è proprio dell'epica. Con Grillo invece l'eccezione (per così dire manierista) si fa norma (per così dire barocca) e il fregio arguto campeggia fin da principio come una sorta di cardine stilistico sul quale poggeranno le molte arguzie spirituali contenute nelle Lagrime. Nell'emblema di Grillo, come si vede, il distico traspone l'affettuoso erotismo della pagana innamorata dalla sfera profana alla sfera del sacro, suggerendo al lettore che l'unica via per accostarsi alla visione del Calvario consisterebbe nel pianto. Le piaghe di Cristo, le uniche materiali, sono espresse dall'immagine. Il testo, invece, parla di piaghe immateriali. La "piaga d'errore" è il peccato che affligge la condizione umana, espresso come "colpa". La "piaga di duol", invece, raffigura metaforicamente le lacrime come l'equivalente mistico del sangue di Cristo: la redenzione dal peccato ("medicina al core"), in altre parole, può essere accolta solo da chi si lasci 'ferire' il cuore dal dolore per ciò che l'immagine cristologica rappresenta. Il paradosso di Armida, che mescola insieme lirica ed epica in un connubio già melodrammatico, diventa insomma, sotto le mani di Grillo, un paradosso teologico, che illumina quello che il cardinale Bellarmino avrebbe definito il bonum lacrymarum, ${ }^{9}$ facendo di quel liquido cerebralmente evocato la spia non solo di un pentimento autentico (su cui si concentrano le Lagrime del penitente), ma anche di un'adesione ai misteri della Croce, il tema cardine dei Pietosi affetti, il ciclo di rime spirituali edito con questo titolo l'anno successivo all'edizione Stigliola delle Lagrime. La compunzione di matrice davidica e neotestamentaria, di conseguenza, viene proposta in limine come il presupposto della contemplazione neotestamentaria della Croce. Grazie all'emblema preliminare, la voce del Penitente che parla nelle Lagrime può essere additata come una sorta di 'figura' (nel senso chiarito da Erich Auerbach $)^{10}$ che troverà il suo 'compimento' nell'io lirico dei Pietosi affetti complementari alle Lagrime (beninteso, non che il nome di Cristo non ricorra nelle Lagrime, ma è solo nel ciclo successivo che la vita e soprattutto la morte di Gesù acquisteranno una funzione strutturale). ${ }^{11}$ Del resto, tipica

9. Il trattato De gemitu columbae sive de bono lacrymarum (1617) è stato riedito alla fine del secolo scorso: Bellarmino 1997.

10. Cfr. Auerbach 1966, pp. 174-221 (Figura, 1944).

11. Due sono le menzioni esplicite di Cristo nelle Lagrime: "Secolo empio e profano! ed è pur vero, / che l'esser oggi imitator di Cristo, / e '1 ricercarlo in umiltà di vita, / quasi s'ascrive a infamia” (III 21, 9-12); "Non rimirar ne' miei peccati rei, / ma nella faccia del tuo Cristo pio; / fra 'l tuo giusto rigore e 'l fallo mio / ponilo, e ti fien cari i pianti miei (IV 20, 1-4)". Dal momento che le Lagrime del penitente non sono comprese nell'ed. Chiarla (Grillo 2013) si citano da Grillo 1629 (pp. 737 e 750-751), anche se i versi suonano identici in Grillo 1594. 
di entrambi i cicli è la spinta a far procedere insieme "affetti" e "concetti", dal momento che questi ultimi sono sentiti e proposti dal monaco-poeta come il veicolo più adeguato all'espressione di una poesia spirituale moderna, tale da mettere le arguzie profane al servizio di un ardito concettismo spirituale. Ben si capisce allora perché un'eroina sentimentale come Armida sia chiamata a nutrire gli affetti devoti del lettore ideale previsto dal monaco. La Gerusalemme liberata, agli occhi di Grillo, aveva per così dire liberato un nuovo tipo di lirica contemporaneamente votata all'arguzia e al pathos.

Che il distico in questione venga a creare un legame speciale tra i due cicli lirici del nostro monaco-poeta trova conferma non solo nel fatto che esso avrebbe introdotto le Lagrime del penitente nell'edizione definitiva del 1629, dove sono proposte come complemento delle cinque parti dei Pietosi affetti, ma anche in un altra sua curiosa epifania. Prima di tornare alla sede originaria, come viatico per il lettore del ciclo di salmi davidici, il distico 'armidiano', infatti, fu provvisoriamente 'delocato' da Grillo e posto come epigrafe interna al ciclo del 1610 intitolato Delle Essequie di Cristo celebrate co 'l pianto di Maria Vergine parte seconda (poi destinato, dal 1613 in poi, a comporre la 'parte quarta' dei Pietosi affetti, tutt'uno con la Parte prima delle Essequie edita nel 1607). A p. 38 del volumetto (Grillo 1610), subito dopo il titolo interno che separa le Essequie propriamente dette dal Pianto di Maria (Pianto di Maria Vergine sopra le orecchie di Cristo estinto), ecco riaffiorare, in modo del tutto inopinato, il distico penitenziale a distanza di sedici anni dall'edizione napoletana della Lagrime (fig. 6). ${ }^{12}$ In questo caso il distico del 1594 da una parte si propone come un madrigale mignon, visto che, anziché da un'immagine, è accompagnato da un argomento che lo denuncia come prosopopea penitenziale: "Il peccator pentito al Crocefisso"; dall'altra esplicita la logica ecfrastica connaturata alla lirica di Grillo in quanto tale, che è solita proporsi come il cartiglio concettoso chiamato a chiosare argutamente un'immagine mistica di natura interiore; e poco conta che il crocefisso sia esplicitato da un'illustrazione o un presupposto iconografico suggerito dalla visione mentale interna al testo. ${ }^{13} \mathrm{Da}$ un certo punto di vista ci si può spingere a affermare che il distico del 1594 non ha neanche bisogno di accompagnarsi a un'immagine materiale e di dar luogo a un emblema, perché l'ispirazione di Grillo, per sua natura, si propone di generare immagini mentali per tramite di concetti poetici.

Per concludere, sembra opportuno segnalare che il distico-esergo di matrice tassiana viene variato a più riprese come espressione arguta di affetti

12. Colgo l'occasione per correggere un'informazione imprecisa (Ferretti 2015, p. 164): il distico del 1594 non ricompare nelle impressioni delle Lagrime successive al 1594, se non, come s'è detto, in quella definitiva del 1629.

13. Cfr. Ferretti 2019b. Sull'elemento paratestuale correntemente detto "argomento", di fondamentale importanza nei libri di rime tra Cinque e Seicento e in qualche modo ecfrastico a sua volta nei confronti del testo che viene a introdurre, sta lavorando Federica Pich con un progetto Humboldt presso la Freie Universität Berlin: Framing the Lyric Subject Matter: Prose Headings in Italian Books of Poetry (c.1450-c.1650); cfr. intanto Pich, 2019. 
penitenziali, sia nel ciclo lirico veterotestamentario, sia in quello neotestamentario. ${ }^{14}$ Si legga il sonetto III 15 delle Lagrime del penitente, che addita in Dio un paradossale "medico" intento a curare le piaghe del peccato con le piaghe delle ingiurie:

Quasi uomo, che non oda, e di pungenti

detti sia privo, io son fatto, o Signore;

così in me serbo il mio giusto rigore,

e gli alti tuoi decreti infra le genti.

Sento le voci ingiuriose, e i denti

della pallida invidia il miser core

mordermi, e segno infausto il proprio onore

all'empio stral delle perverse menti.

Ma son, Medico mio, queste l'amare

medicine onde purghi, e i fochi e i ferri,

ond'incidendo, e ardendo i morbi sani;

ché tu le piaghe con le piaghe sani,

e coi peccati i peccator fai sani,

onde mi son cotante ingiurie care. ${ }^{15}$

Oppure si veda il madrigale $A$ h, con mediche voci interna piaga (I 38) edito per la prima volta nei Pietosi affetti del 1595 tutt'uno con un argomento che, anziché al curatore Pietro Colelli da Sessa, andrà sicuramente attribuito a Grillo stesso (Non potersi saldar la piaga d'errore senza la piaga di dolore): ${ }^{16}$
Ah, con mediche voci interna piaga
lunga stagione aperta
mal si risana, e chiude,
sì l'arme son, che l'han ferita, crude,
se di novo, GIESÙ mio, non l'impiaga
duol di vederti in questa Croce esangue,
e non stagni nel cor sangue, con sangue. ${ }^{17}$

Un madrigale che si segnala non solo per essere una perfetta variazione concettuale del distico dal quale abbiamo preso le mosse, ma anche per il trapianto

14. Aggiungo, per scrupolo, che nell'ed. definitiva delle Rime morali (Grillo 1599, p. 49r), la ripresa del modello tassiano è solo parziale, ma è comunque significativa perché il poeta qui si auto-rappresenta come un pittore intento ritrarre i concetti celestiali frutto delle proprie contemplazioni: "ciò che mi dettan qui, ciò che contempio / là su, mi ingegno di ritrar in carte / per farne dolce medicina al core" (sonetto n. 119: Al sepolcro m'avezzo e ogn'or co' morti).

15. Si cita da Grillo 1629 (ma anche in questo caso Grillo 1594 non differisce).

16. Nella 'parte prima' dei Pietosi affetti di inizio secolo (Grillo 1601, p. 23), che accresce il corpus Grillo 1595, si legge addirittura, con ridondanza, un doppio argomento "Chiede a sanar piaga d'errore piaga di dolore. // Non potersi saldar la piaga d'errore senza la piaga di dolore" (ridondanza corretta in Grillo 1629).

17. Grillo 2013, p. 108 (mio il corsivo). Il testo del madrigale e del suo argomento non differiscono in Grillo 1595 (pp. 16 e 214), dove erano apparsi per la prima volta. 
devoto e sorprendente di elementi patetici 'rubati' al Tasso lirico sublime; in questo caso si tratta dei vv. 21-26 (e ancora una volta si evoca una piaga...) della Canzone al Metauro, a stampa dal 1582:

Oimè! dal dì che pria trassi l'aure vitali e i lumi apersi in questa luce a me non mai serena, fui de l'ingiusta e ria trastullo e segno, e di sua man soffersi piaghe che lunga età risalda a pena. ${ }^{18}$

Oppure si veda il sonetto I 408, il cui argomento "Invita gli occhi a piangere, et a porger co 'l pianto medicina al cuore" e il cui incipit (Occhi piangete e medicate il core) riscrive in senso spirituale l'analogo attacco petrarchesco (Occhi piangete: accompagnate il core, RVF 84), sviluppando ancora una volta il concetto di espiazione come medicina dell'anima (anche ai vv. 12-13: "e di sua colpa / sia medicina il duol" ${ }^{19}$. $\mathrm{O}$ infine - ma è evidente che una rassegna di questo tipo non ha alcuna pretesa di sistematicità - si veda il madrigale ecfrastico (I 322) ${ }^{20}$, composto per un dipinto devozionale di provenienza veneziana ("Sopra un'imagine della Maddalena di man di Tiziano"), ossia sullo stesso soggetto che dà materia alle ottave di Marino comprese nella Galeria (Historie 37b):
Questa è colei (ben io la riconosco a quell'affisso essangue, de la cui vista innamorata langue) che peccatrice, e poi pentita, il tosco mortal versò per gli occhi in largo umore, e ne fe' santa medicina al core; è d'essa certo, e qui piangendo tira a pianger chi la mira, per miracolo sol d'alto pittore; se ben per opra d'invida natura altrui sembra pittura.

Né importa qui stabilire se di un vero Tiziano (l'“alto pittore") si trattasse, ${ }^{21}$ visto che, ancora una volta, il lettore di Grillo è chiamato a concentrarsi con occhi bagnati sulla pittura metaforica e obliqua che questo tipo di lirica ingegnosa viene a produrre nel teatro della mente, secondo una logica ecfrastica che era già tutta in nuce nel distico 'armidiano' del $1594 .^{22} \mathrm{Il}$ peccato adombrato

18. T. Tasso, Rime, n. 573. Si cita il testo Solerti-Maier da Tasso 1994 (mio il corsivo).

19. Questo sonetto (Grillo 2013, p. 300) è invece anteriore all'emblema del 1594, visto che compare già come n. 71 nella Parte seconda di Rime spirituali (Grillo 1989, p. 28r).

20. Grillo 2013, p. 261 (aggiunto al corpus dei Pietosi affetti in Grillo 1601, p. 207).

21. Sulle repliche e sulle varianti delle Maddalene tizianesche, cfr. Rearik 2001; Tagliaferro \& Aikema 2009, pp. 249-258.

22. Degna di nota, infine, è la fortuna secentesca del v. 1 del distico in esame, riportato alla sua matrice erotica tassiana, a metà secolo, dal veneziano Giovanni Battista Bertanni, La ninfa 
nel distico finale, a sua volta, coincide con la renitenza a piangere di un osservatore freddo e incapace di lasciarsi commuovere dal concetto poetico: un osservatore che, anziché ammirare una finzione che sembra realtà ("è d'essa certo"), mosso da "invida natura" preferisce non lasciarsi ingannare né dal quadro di Tiziano (solo "pittura", e non più realtà), né dall'evidenza metafisica di Grillo stesso, che col pittore sta competendo.

\section{Bibliografia}

Alciato, A. (2009). Il libro degli emblemi (secondo le edizioni del 1531 e del 1534). Introduzione, traduzione e commento di M. Gabriele. Milano: Adelphi.

Ammirato, S. (1562). Il Rota overo dell'imprese. Napoli: Scotto.

Arbizzoni, G. (2002). "Un nodo di parole e di cose". Storia e fortuna delle imprese. Roma: Salerno.

Auerbach E. (1966). Studi su Dante, trad. it. Milano: Feltrinelli.

Bellarmino, R. (1997). Scritti spirituali (1615-1620), II (Il gemito della colomba. Le sette ultime parole di Cristo), coordinamento di P. Giustiniani, introduzione di G. Galeotta S.J. Brescia: Morcelliana.

Bertanni, G. B. (1642). La ninfa spensierata. Padova: Cadorino.

Bertanni, G. B. (2011). Con la sampogna in man, col flauto al labro: edizioni commentate della "Ninfa guerriera" di Giovan Battista Lampugnano e della "Ninfa spensierata" di Giovan Battista Bertanni (a cura di Simona Bortot). Bologna: Archetipolibri.

De Blasi N. (1976). Bertanni, Giovanni Battista. In Dizionario biografico degli Italiani (vol. 9, pp. 460-461). Roma: Istituto dell'Enciclopedia Italiana. Disponibile su: https://www.treccani.it/enciclopedia/giovanni-battista-bertani_resec4df813-87e7-11dc-8e9d-0016357eee51_\%28Dizionario-Biografico\%29/

Ferretti F. (2012). Le Muse del Calvario. Angelo Grillo e la poesia dei benedettini cassinesi. Bologna: il Mulino.

Ferretti, F. (2015). L'ingegnoso penitente. Angelo Grillo e i Salmi penitenziali. In R. Alhaique Pettinelli, R. Morace, P. Petteruti Pellegrino \& U. Vignuzzi (Edd.), La Bibbia in poesia. Volgarizzamenti dei Salmi e poesia religiosa in età moderna [Numero monografico]. Studi (e testi) italiani, 35, 151-168.

Ferretti, F. (2019a). Epica inclusiva. Intersezioni di genere nella "Liberata". In G. Barucci, S. Carapezza, M. Comelli, C. Zampese (Edd.), "D'otto in otto versi". Il poema in ottave come ricettore di generi (pp. 73-88). Firenze: Franco Cesati.

spensierata, favola pastorale del 1642 rappresentata dall'Accademia padovana dei Disuniti. Si dà qui lettura di un carme epigrammatico, "sani piaga mortal piaga d'errore", che viene poi corretto in modo meno cruento: "sani piaga di duol piaga d'errore" (Bertanni 1642, pp. 31-32, atto I, scena IV). La favola in questione, alla quale la sorte non ha arriso neppure nel suo secolo, è stata riedita in tempi recenti da Simona Bortot (Bertanni 2011), alla quale si deve un'introduzione che tratta il contributo di Bertanni - e del milanese Lampugnano - all'interno della linea culturale promossa dall'Accademia veneziana degli Incogniti di Loredan. La scena contenente il "furto" ai danni di Grillo si legge qui alle pp. 249-250, ma nel commento non si fa menzione del monaco. Per un profilo dell'attività letteraria di Bertanni, cfr. anche De Blasi 1976. 
Ferretti F. (2019b). L'ecfrasi mistica di Angelo Grillo. In A. Campana, F. Giunta \& E. Ripari (Edd.), Fra norma e obbedienza. Letteratura e immagini sacre in Italia nell'epoca della Controriforma (pp. 111-122). Bologna: I libri di Emil di Odoya.

Giraud, Y. (1982), L'emblème à la Renaissance. Sedes: Paris.

Grillo, A. (1589), Rime [Parte prima di Rime morali \& Parte seconda di Rime spirituali]. Bergamo: Comino Ventura.

Grillo, A. (1593). Lagrime del penitente ad imitatione de' sette salmi penitentiali di Davide. Bergamo: Comino Ventura.

Grillo, A. (1594). Lagrime del penitente ad imitatione de' sette salmi penitentiali di Davide. Napoli: Stigliola.

Grillo, A. (1595). Pietosi affetti. Genova: Bartoli.

Grillo, A. (1596). Pietosi affetti. Vicenza: Perin Libraro.

Grillo, A. (1599), Rime [Rime morali \& Pompe di morte]. Venezia: Ciotti.

Grillo, A. (1601). Pietosi affetti et Lagrime del penitente. Venezia: Ciotti.

Grillo, A. (1610). Dell'Essequie di Cristo celebrate co 'l Pianto di Maria Vergine parte seconda. Venezia: Giunti e Ciotti.

Grillo A. (1629). Pietosi affetti [in cinque parti: 1. Pietosi affetti; 2. Pietosi affetti. Parte seconda; 3. Cristo flagellato; 4. Essequie di Cristo. Parti prima e seconda; 5. Aggiunta (con un'aggiunta ulteriore di testi sulla liquefazione del sangue di san Giovanni Battista); Lagrime del Penitente], Venezia: Deuchino.

Grillo A. (2013). Pietosi affetti (a cura di M. Chiarla). Lecce: Argo.

Leri, C. (1994). Sull'arpa a dieci corde. Traduzioni letterarie dei salmi 1641-1780. Firenze: Olschki.

Leri, C. (2011). "La voce dello Spiro". Salmi in Italia tra Cinque e Settecento. Alessandria: Edizioni dell'Orso.

Morace, R. (2015). I Salmi tra Riforma e Controriforma. In R. Alhaique Pettinelli, R. Morace, P. Petteruti Pellegrino \& U. Vignuzzi (Edd.), La Bibbia in poesia. Volgarizzamenti dei Salmi e poesia religiosa in età moderna [Numero monografico]. Studi (e testi) italiani, 35, 55-81.

Morace, R. (2018). La Giolitina "Salmi penitentiali”. Edizioni e ristampe. Linguistica e letteratura, 42 (1-2), 37-62. doi: https://doi.org/10.19272/201701602002.

Piatti A.A. (2007). "E l'uom pietà da Dio, piangendo, impari". Lacrime e pianto nelle rime sacre dell'età del Tasso. In C. Delcorno \& M. L. Doglio (Edd.), Rime sacre tra Cinquecento e Seicento (pp. 53-106). Bologna: Il Mulino;

Piatti A.A. (2010) "Su nel sereno de' lucenti giri”. Le Rime sacre di Torquato Tasso. Alessandria: Edizioni dell'Orso.

Pich, F. (2019). On the Threshold of Poems: a Paratextual Approach to the Narrative/ Lyric Opposition in Italian. In F. Venturi (Ed.), Self-Commentary in Early Modern European Literature, 1400-1700 (pp. 99-134). Leiden-Boston: Brill. doi: https:// doi.org/10.1163/9789004396593_006

Pietrobon E. (2019). La penna interprete della cetra. I "Salmi» in volgare e la poesia spirituale italiana nel Rinascimento, Roma: Edizioni di Storia e Letteratura. Disponibile su: http://bitesonline.it/wp-content/uploads/2020/06/Bites013_Pietrobon_interni.pdf

Praz, M. (2014). Studi sul concettismo. Emblema, impresa, epigramma, concetto, con uno scritto di M. Maggi. Milano: Abscondita

Quondam, A. (2005). Note sulla tradizione della poesia spirituale e religiosa (parte prima) - Saggio di bibliografia della poesia spirituale (1471-1600). In A. Quondam (Ed.), Paradigmi e tradizioni (pp. 127-282). Roma: Bulzoni. 
Rearik W. R. (2001). Le "Maddalene penitenti” di Tiziano, Arte veneta 58, 22-41.

Ricci, S. (2019). Stigliola, Nicola Antonio. In Dizionario biografico degli italiani (vol. 94, pp. 246-249). Roma: Istituto dell'Enciclopedia Italiana. Disponibile su: https://www.treccani.it/enciclopedia/nicola-antonio-stigliola_\%28DizionarioBiografico\%29/.

Tagliaferro, G. \& Aikema, B. [con Mancini, M. \& Martin, A. J., redazione: T. Vecchi] (2009). Le botteghe di Tiziano, Firenze: Alinari 24 Ore, pp. 249-258.

Tasso, T. (1994). Rime (a cura di B. Basile). Roma: Salerno ed.

Tasso, T. (2009). Gerusalemme liberata (a cura di F. Tomasi). Milano, Rizzoli-Bur, 2009. Torre, A. (2012). "Purgar con gli occhi il fallo della lingua". Eloquenza visuale delle "Lacrime di san Pietro". In E. Ardissino \& E. Selmi (Edd.), Visibile teologia. Il libro sacro illustrato in Italia tra Cinque e Seicento (pp. 323-344). Roma: Edizioni di Storia e Letteratura.

Turchi, F. (1572). Salmi penitentiali di diversi eccellenti autori. [...] Scelti dal reverendo p. Francesco da Trivigi carmelitano. Venezia, Giolito.

Turchi, F. (2016). "Salmi penitenziali di diversi eccellenti autori" [Giolito 1568]. Introduzione e testo critico a cura di R. Morace, Pisa: ETS.

Zaja, P. (2013). Salmi e lirica volgare nel Cinquecento. In Gibellini, P. (Dir.), La Bibbia nella letteratura italiana, vol. V. Melli, G. \& Sipione M. (Edd.), Dal Medioevo al Rinascimento (pp. 549-568). Brescia: Morcelliana.

Zaja, P. (2014). Francesco Turchi e i "Salmi penitentiali, di diversi eccellenti autori" (Venezia, 1568), Quaderni veneti», 3, 65-73.

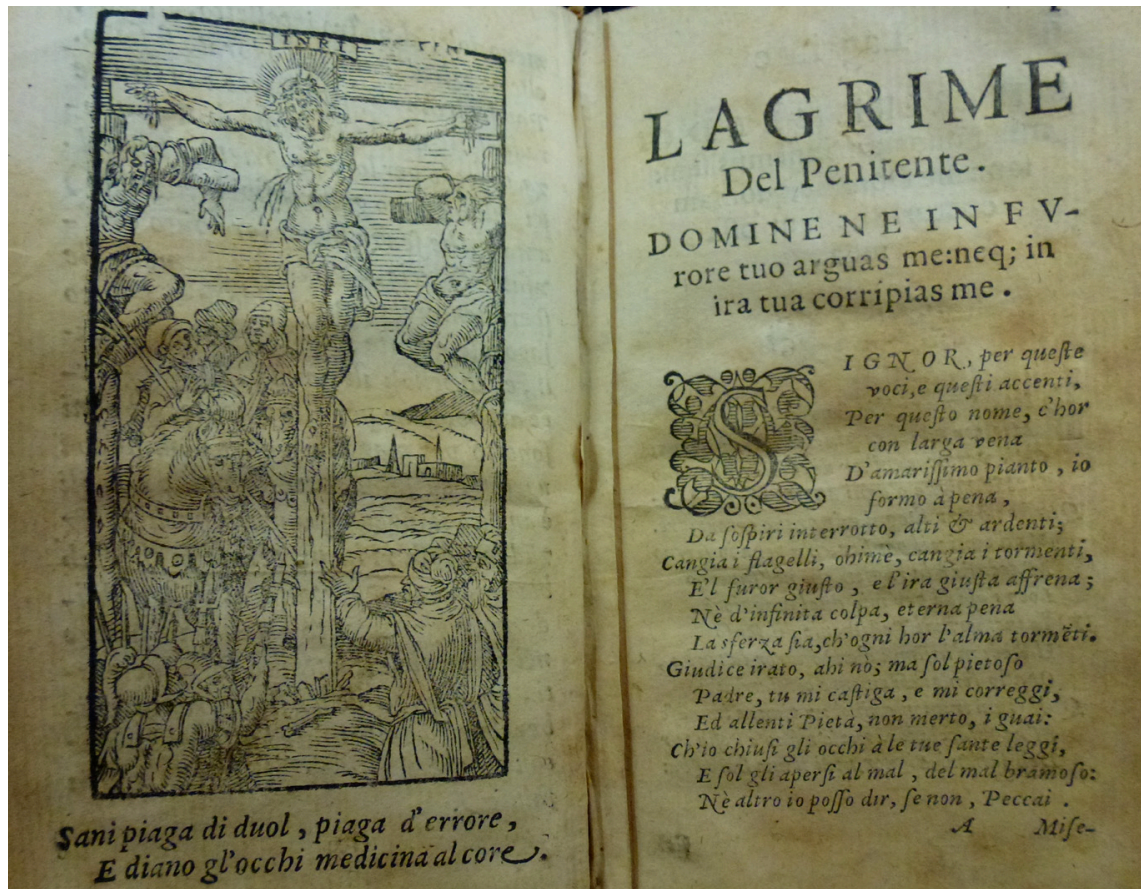

Fig. 1. Crocefissione e distico penitenziale (Grillo 1594). 


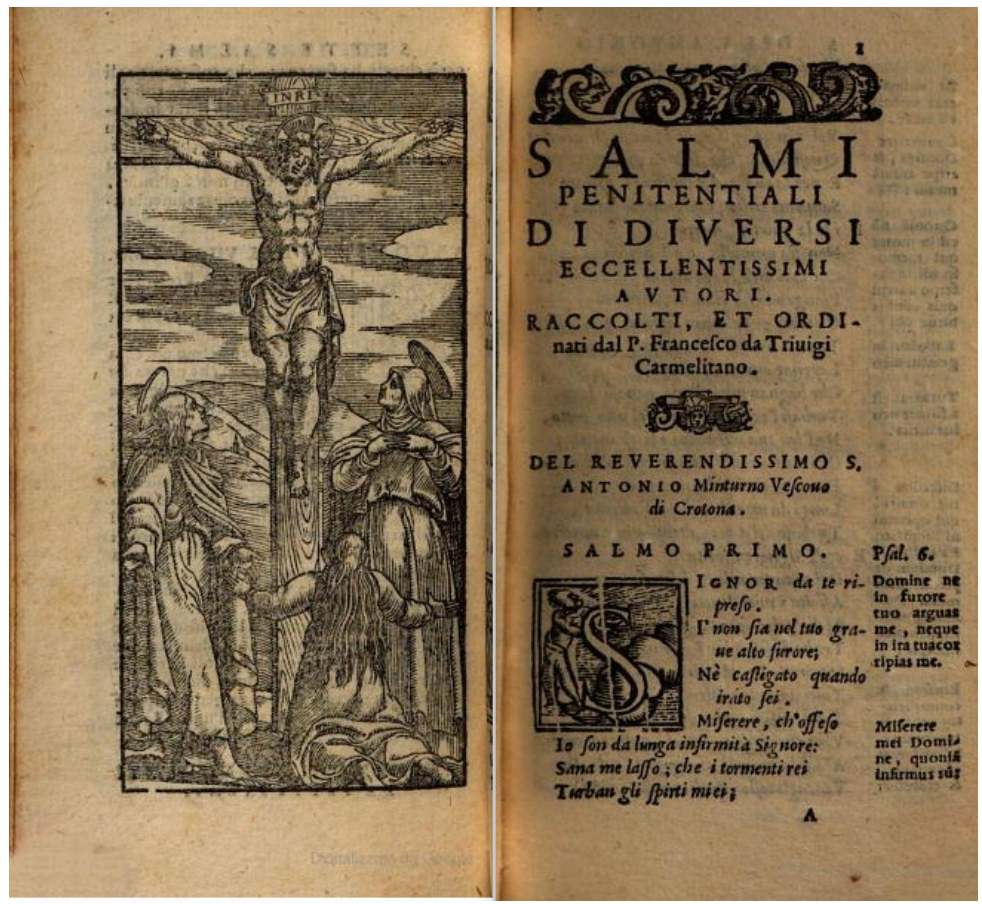

Fig. 2. Cristo in croce tra dolenti (Turchi 1572).

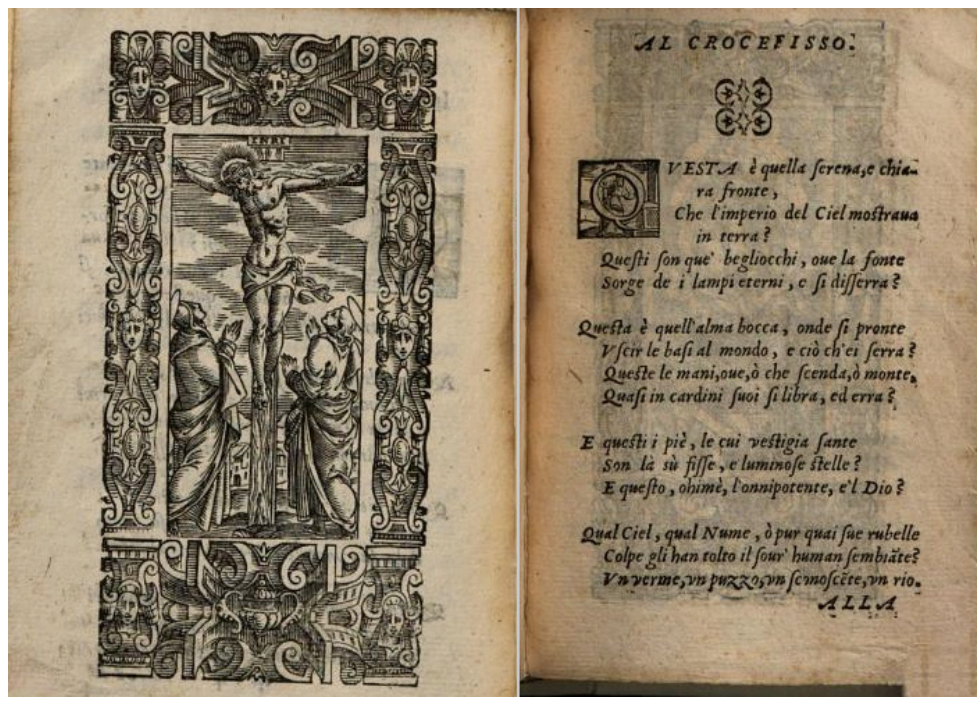

Fig. 3. Cristo in croce tra dolenti (Grillo 1595). 


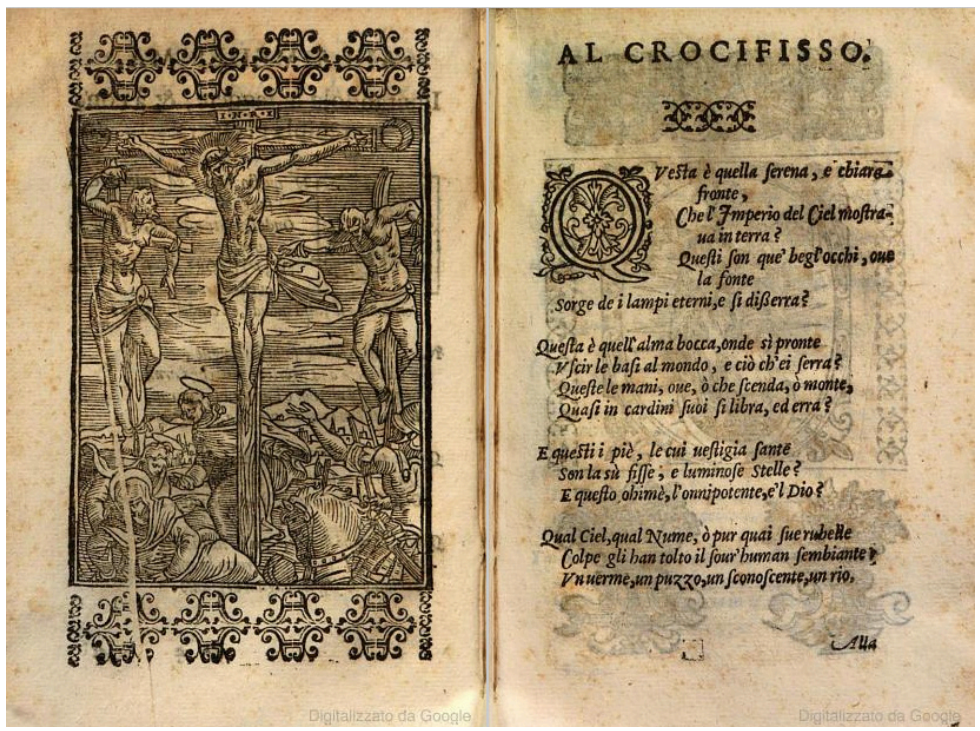

Fig. 4. Crocefissione (Grillo 1596).

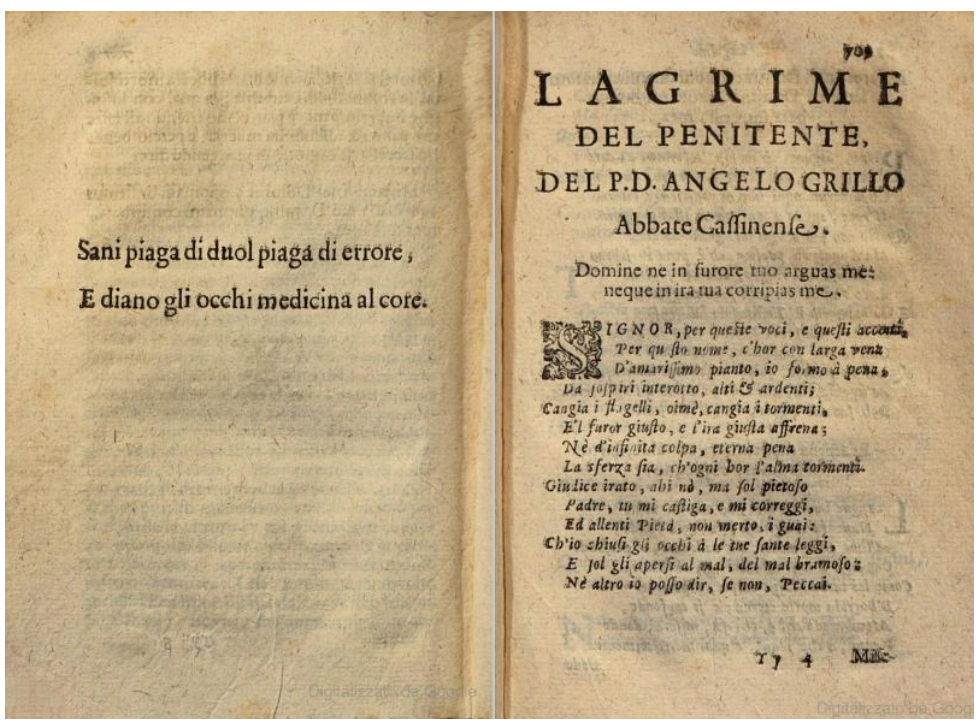

Fig. 5. Distico penitenziale (Grillo 1629). 


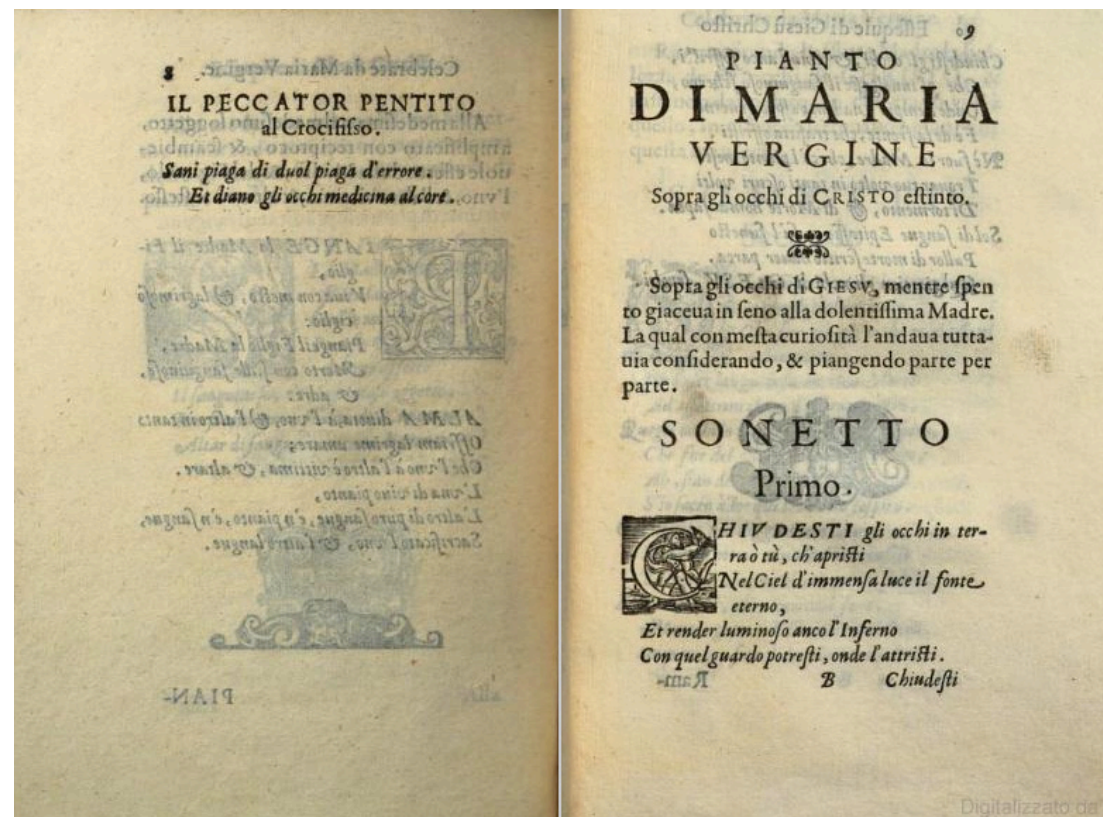

Fig. 6. Distico penitenziale (Grillo 1610). 
